\title{
Local Action of Intrahypophyseal Implants of Estrogen as Revealed by Staining with Peroxidase-labeled Antibody '
}

\author{
BRIAN E. GERSTEN AND BURTON L. BAKER \\ Department of Anatomy, The University of Michigan Medical School, \\ Ann Arbor, Michigan 48104
}

\begin{abstract}
The direct action of $17 \beta$-estradiol and cholesterol on cells of the pituitary pars distalis, as revealed by staining with peroxidase-labeled antibody, was studied in female rats. Pellets of pure cholesterol and of estradiol mixed with choles. terol were implanted into the left lobe of the pars distalis 14-32 days after ovariectomy and left in place for 7-16 days. Rabbit antisera to rat prolactin, human growth hormone, human chorionic gonadotropin (for gonadotropes - presumably luteinizing hormone cells), and porcine corticotropin were used. In no case were cells altered in the contralateral lobe of the pars distalis; cholesterol likewise had no significant effect on the ipsilateral lobe. However, in the ipsilateral lobe containing an estrogen pellet, prolactin cells were hypertrophied and hyperplastic; cells assumed to be responsible for luteinizing hormone secretion were reduced in size and stained more intensely; and growth hormone cells were reduced in size. Corticotropin cells remained unaffected. For the most part estrogenic effects were distributed ventrally, caudally and laterally from the pellet and not far medially, never reaching the midline. It was concluded that estrogen acts directly on the hypophysis, the spread of the effects reflecting the direction of blood flow within the gland. These observations support the hypothesis that the pituitary gland serves as a site for feedback action by estrogen.
\end{abstract}

One of the most important current problems in neuroendocrinology is to ascertain whether the hypothalamus or hypophysis serves as the primary site for feedback action by target-organ hormones. Evidence available at present indicates that both sites are probably responsive to estrogen but the manner in which the functional roles of the hypothalamus and hypophysis are integrated remains unclear.

Several investigators have implanted ovarian tissue or estrogen into the hypothalamus and, from the effects observed, concluded that estrogens act primarily on the hypothalamus. Thus, Flerkó and Szentágothai ('57) found that ovarian secretory activity was depressed in rats bearing small intrahypothalamic autotransplants of ovarian tissue, but not in rats bearing intrapituitary implants. Likewise, implantation of estrogen into the median eminence of the rabbit caused depression of ovarian function (Lavidson and Sawyer, '64), lowered pituitary luteinizing hormone content (Kanematsu and Sawyer, '63c, '64), suppressed the postovariectomy increase in size and number of gonadotropic basophils (Kanematsu and Sawyer, '63b), and elevated the prolactin content of the gland (Kanematsu and Sawyer, '63a). These workers found that estrogen placed into the hypophysis failed to duplicate these effects, the pituitary prolactin content actually being depressed (Kanematsu and Sawyer, 63a).

Apparently the rat responds differently to these experimental procedures. After Lisk ('60) showed that estrogen implanted into the hypothalamic arcuate nucleus causes atrophy of the genital tract, McCann and his colleagues found that estrogen located in either the median eminence or hypophysis was effective; such implants prevented the postovariectomy rise in plasma luteinizing hormone (Ramirez Abrams and McCann, '64) and elicited peripheral effects indicative of accelerated prolactin secretion (Ramirez and McCann, '64; Chowers and McCann, '67). These investigators concluded that estrogen exerts its feedback action either in the hypothalamus or directly on the hypophysis. Also, in prepubertal rats estrogen induced ovarian

\footnotetext{
1 Supported in part by research grants from the USPHS (HD 03159-02) and from the University of Michigan Horace $H$. Rackham School of Graduate Studies.
} 
luteinization more effectively when placed into the hypophysis than into the hypothalamus (Döcke and Dörner, '65).

More information concerning the direct action of estrogen on hypophyseal cytology should indicate the capacity of estrogen to elicit feedback responses at the pituitary level. Since the effect of intrapituitary estrogen on the structure of pituitary cell types has been revealed clearly for only gonadotropic basophils considered as a class (Bogdanove, '63), this study was carried out to examine the responses of cells producing prolactin, growth hormone, corticotropin, and those that probably secrete luteinizing hormone as revealed by immunochemical staining with peroxidaselabeled antibody.

\section{MATERIALS AND METHODS}

Young adult Sprague-Dawley rats were ovariectomized one, two, or four weeks prior to pellet placement to allow involution of prolactin cells and the development of postovariectomy changes in basophils. The number of animals in each group is shown in table 1.

Steroid pellets were prepared according to the procedure employed by Bogdanove ('63). An estrogen-cholesterol mixture was prepared by dissolving $0.5 \mathrm{mg}$ of $17 \beta$-estradiol benzoate with $100 \mathrm{mg}$ of cholesterol in $4 \mathrm{ml}$ of ether, the ether subsequently being evaporated off. The mixed crystals were heated to the melting point, after which the end of a 21-gauge needle was inserted into the melted sterols for about one minute. The needle was then withdrawn, cooled, and the pellet extruded. The estrogen dilution of $1: 200$ is somewhat less than the maximal dilution of 1:500 which Bogdanove ('63) found effective in suppressing castration cell formation. Pellets of pure cholesterol were also made.
For implantation a pellet less than one $\mathrm{mm}$ long of either the estradiol mixture or cholesterol was inserted into a blunted 20gauge spinal needle. The pellet was implanted stereotaxically into the left pars distalis according to the coordinates of DeGroot ('59), the needle being lowered to the sphenoid bone and raised prior to extrusion. The needle regularly entered the left lobe of the pars distalis just lateral to the pars intermedia. The retropharyngeal approach commonly employed for hypophysectomy was also used to place other pellets in or in contact with the inferior surface of the left pituitary lobe. The retropharyngeal approach eliminated two deficiencies that attend use of the stereotaxic method, namely damage to the brain and possible deposition of estrogen along the needle track.

The animals were killed one or two weeks after the pellets were implanted, and the hypophyses were examined in situ for hypertrophy and location of the pellet. They were fixed in Bouin's fluid, embedded in paraffin and sectioned serially in a dorsoventral plane at $3 \mu$. Many sections were stained with the Masson trichrome procedure. Other sections, representative of different zones through the gland, were stained immunochemically for prolactin cells, growth hormone cells, corticotropin cells, and gonadotropes. For immunochemical staining the method of Nakane and Pierce ('67) was used. It consists of the following essential steps. First, rabbit antiserum to the hormone is applied to a pituitary section. Second, sheep antiserum to rabbit gamma-globulin conjugated with peroxidase is applied. Finally, the section is stained histochemically with 3,3'-diaminobenzidene serving as substrate for the demonstration of peroxidase, the reaction

TABLE 1

Numbers of rats subjected to experimental procedures

\begin{tabular}{|c|c|c|c|c|c|}
\hline \multicolumn{2}{|c|}{ Duration of treatment (days) } & \multicolumn{4}{|c|}{ Number of rats } \\
\hline \multirow{2}{*}{$\begin{array}{l}\text { Ovariectomy to } \\
\text { termination }\end{array}$} & \multirow{2}{*}{$\begin{array}{c}\text { Implantation to } \\
\text { termination }\end{array}$} & \multicolumn{2}{|c|}{ Stereotaxic implantation } & \multicolumn{2}{|c|}{ Retropharyngeal implantation } \\
\hline & & Estrogen & Chalesterol & Estrogen & Cholesterol \\
\hline 14 & 7 & 4 & - & - & - \\
\hline 21 & 7 & 3 & 2 & - & - \\
\hline 21 & 14 & 10 & 8 & - & - \\
\hline $28-32$ & $11-16$ & 23 & 12 & 11 & 2 \\
\hline
\end{tabular}


product delineating the cells that contain the hormonal antigen.

The following hormonal antigens were used: rat prolactin (HIV-8-C) prepared by S. Ellis, human growth hormone (Raben, lots 12 and 14), porcine corticotropin, and human chorionic gonadotropin. Additional information on preparation of the antisera and specificity of the staining procedure has been published for prolactin, growth hormone (Baker et al., '69) and corticotropin (Baker et al., in press) cells. Monroe and Midgley ('69) have described the preparation of the antiserum to human chorionic gonadotropin (HCG) and its usefulness in staining luteinizing hormone cells in the rat by the immunofluorescent procedure. The antiserum employed in this study had comparable immunological properties (personal communication). Even though the cells demonstrated were most numerous at the periphery of the gland, this distribution being characteristic of luteinizing hormone cells as described by Monroe and Midgley, the specificity of the anti-HCG for differentiating luteinizing hormone from follicle-stimulating hormone cells was not verified in the present study. Therefore, the cells revealed by the use of anti-HCG are designated gondadotropes.

\section{OBSERVATIONS}

For several reasons immunochemical staining proved superior to histologic staining for study of the local action of estrogen on the hypophysis. First, when the corticotropin cell and other chromophils were small and inactive they could not be identified accurately in Masson-stained preparations. Second, it was difficult to evaluate a general change in number and size of a single cell type throughout a Massonstained section when all other cells were colored also. Third, compression of the gland during excision interfered with subsequent histologic staining but was somewhat less troublesome if immunochemical procedures were followed. In the subsequent description of the effects of the intraphypophyseal implantation of estrogen pellets, observations made with immunochemical staining will be emphasized. The Masson-stained preparations were especially useful for analysis of the extent and distribution of the estrogen effects.
Prolactin cells. Following ovariectomy prolactin cells undergo marked reduction in size (Baker et al., '69). In the present study this response was evident 14 days after ovariectomy but was more pronounced after longer postoperative periods. Cholesterol pellets implanted into the pars distalis by either operative procedure did not cause any change in prolactin cells (figs. 1, 2).

In glands implanted with estrogen pellets, prolactin cells were greatly enlarged as compared with those in ovariectomized controls, and contained more large secretory granules; the nucleus and Golgi area were hypertrophied (figs. 3, 5). These changes were present at seven days after implantation but were more pronounced at 14 days. Occasional prolactin cells contained mitotic figures, this hyperplasia combined with prolactin cell enlargement accounting for the unilateral hypertrophy of the ipsilateral lobe of the pars distalis that was observed in some cases (fig. 3 ).

The stimulating effect of estrogen on prolactin cells was restricted to the ipsilateral side; in no case were prolactin cells altered on the contralateral side (fig. 4). Furthermore, only a portion of the ipsilateral lobe was affected, the amount of tissue involved depending chiefly on the location of the pellet. The extent of the tissue affected was greatest for pellets located rostrodorsally and was progressively less as the pellet was located more caudally and ventrally. The effect extended only a short distance rostrally and did not involve the rostral margin of the gland unless the pellet was located near it. Medially, spread of the effect was markedly limited and did not reach the midline (figs. $3,6,7,9$ ) unless the pellet was located there. The medial border of the responsive zone was sharply demarcated (figs. 6,9). Spread of the effect toward the dorsal surface of the gland was quite variable following stereotaxic implantation. When the pellet was located in the middle or ventral region of the lobe, enlargement of prolactin cells extended to the dorsal surface in many cases (fig. 3). However, one could never be certain that fragments of estrogen had not been inadvertently implanted along the needle track. In other cases a zone along 
the dorsal margin remained unaffected. Spread of the effect toward the dorsal surface from pellets placed inferior to the gland by the retropharyngeal approach was clearly limited (fig. 7).

The zone of prolactin cell enlargement always extended to the lateral tip of the pars distalis when the pellet was implanted stereotaxically and located within the substance of the gland. When the pellet was placed inferior to the gland, the lateral spread was quite limited (figs, 7,8 ). In summation, the major distribution of the effect was caudal, lateral and ventral to the pellet. Pellets located rostrally stimulated prolactin cells at the caudal margin of the gland. In such cases the effect always reached the lateral and ventrolateral margins of the gland, with the medial extent of the spread becoming progressively less toward the caudal margin of the gland.

The direction of distribution of estrogen from pellets placed above the gland is indicated by observations on several cases. In one rat, the pellet was found at autopsy to be in the left portion of the median eminence and the absence of a needle track in the hypophysis indicated that the needle had not reached the gland. In this case, prolactin cells were enlarged throughout the left lobe of the pars distalis and slightly across the midline into the right lobe (figs. 10,12). Prolactin cells remained unchanged throughout the right lobe. In three other cases the estrogen pellet was shown by gross inspection, or in microscopic sections, to be located above and in contact with the left lobe of the pars distalis with no needle track being found in the gland. In these cases prolactin cell enlargement had occurred through a zone extending from the dorsal surface for a variable distance into the gland, reaching the ventral surface only in the caudal region. The medial extent of the response varied from the middle of the lobe to within a short distance from the midline. In all of these cases, the region of responsivity extended to the lateral tip.

Growth hormone cells. Cholesterol pellets elicited no change in growth hormone cells (figs. 13, 14). On the other hand, estrogen pellets induced two changes in growth hormone cells. The cells were more widely dispersed in the ipsilateral lobe of the pars distalis (figs. 15, 17) than in the contralateral lobe (fig. 16), at least in part, this effect being secondary to hypertrophy and multiplication of the intervening prolactin cells. Also, growth hormone cells were generally somewhat smaller (fig. 17) in the neighborhood of the estrogen pellet than on the contralateral side (fig. 16) or in the cholesterol-implanted gland. The distribution of these changes was similar to that for alterations in prolactin cells, likewise extending for a limited distance toward the midline (fig. 18).

Corticotropin cells. Comparison of the size and distribution of corticotropin cells on the ipsilateral and contralateral sides of cholesterol-implanted hypophyses revealed no effect of the steroid (figs. 19, 20). Estradiol induced some secondary dispersal of corticotropin cells (fig. 21) in the vicinity of the pellet (figs. 23, 24), as compared with the contralateral side (fig. 22). There was no consistent change in the morphology of corticotropin cells although certain of the cells close to the pellet sometimes appeared smaller.

Gonadotropes. Although figure 25 suggests that cholesterol may have reduced the number of gonadotropes per unit area above and lateral to the pellet, this was not generally the case. However, estrogen pellets caused a reduction in size of these cells with an increase in the density and intensity of immunochemical staining of the cytoplasm (figs. 27,29 ) as compared with gonadotropes on the contralateral side (fig. 28 ) and in the cholesterol-implanted gland (fig. 26). These effects occurred dorsal and lateral to the pellet but only for a short distance on the medial side (fig. 30). Some specimens suggested a compensatory hypertrophy of gonadotropes on the contralateral side of the estrogen-implanted gland (fig. 28) when compared with cells in the cholesterol-implanted gland (fig. 26). Gonadotropes averaged about $14 \mu$ in largest diameter near estradiol pellets, $18 \mu$ in cholesterol-implanted glands, and $22 \mu$ in the contralateral lobe of estradiol-implanted glands. The intraglandular distri bution of estrogen-induced modifications in gonadotropes was similar to that for prolactin cells. When the estrogen pellet was 
located in the median eminence on one side, similar changes occurred in the ipsilateral lobe of the pars distalis but were less intense than when the pellet was in the gland itself.

\section{DISCUSSION}

\section{Correlation of cytology with functional changes induced by estrogen}

Prolactin cells. The capacity of estrogen to increase the glandular content and secretion of prolactin is established (Meites and Nicoll, '66). These responses have been correlated with hypertrophy and hyperplasia of a special class of acidophils (luteotropes) as revealed by histologic staining and by electron microscopy (Purves, '61). Immunochemical staining confirms these conclusions and permits a better appreciation of the magnitude of the response by revealing more clearly the large number of prolactin cells involved, particularly when they are small and inactive.

Growth hormone cells. Little attention has been given to the influence of estrogen on growth hormone cells. However, on the basis of chromatographic and immunoassay analysis of the hypophysis, it appears that systemically administered estrogen tends to lower the concentration and possibly the total content of growth hormone in the gland (literature reviewed by Baker, in press), although some evidence suggests that this effect may be mediated in part by a caloric deficiency (Birge et al., '67). The reduction in size and possibly lowered incidence of growth hormone cells elicited in this study by estrogen implants indicate that estrogen has the potential to suppress growth hormone cells directly.

Corticotropin cells. The influence of estrogen on the structure of corticotropin cells has not been studied previously under conditions where their identity was established unequivocally. Since the content of corticotropin in female rat hypophyses (Kitay, '63) and concentration in the plasma (Fonzo et al., '67) may be increased by systemic treatment with estrogen, enlargement of corticotropin cells might have been expected. The reason for the negative result is not evident. Estrogen implanted into the rat hypophysis increases adrenal weight (Chowers and Mc-
Cann, '63) and concentration of corticosteroids in blood and adrenal gland (Richard, '65), indicating that corticotropin secretion is stimulated.

Gonadotropes. The secretion of folliclestimulating and luteinizing hormones by basophils, their enlargement after ovariectomy and regression under estrogen treatment have been established (Purves, '61). Thus, the reduction in size of gonadotropes observed in this study around pellets of estrogen corresponds with effects described previously after systemic administration and intrapituitary implantation of the hormone (Bogdanove, '63). However, clarification of the cellular source(s) of FSH and $\mathrm{LH}$ and possible differential responses to estrogen require further study since Nakane (in press) now holds that one cell type produces both hormones on the basis of observations made with immunochemical staining.

\section{Neuroendocrine implications}

Several deductions that pertain to the present understanding of the hypothalamohypophyseal relationship may be drawn from these observations.

Direct stimulation of the hypophysis. The first is that estrogen has the capacity to stimulate the pars distalis in situ by direct action since enlargement and hypertrophy of prolactin cells are localized to the region of the hormone pellet. The increased relative percentage of acidophils reported by Kanematsu and Sawyer ('63b) in rabbits bearing intrapituitary estrogen implants is probably accounted for by direct stimulation of prolactin cells, since they observed hypertrophy of only "some acidophils." The demonstrated acceleration of prolactin secretion by addition of estrogen to a medium in which the rat pars distalis is cultured (Nicoll and Meites, '62) also supports this conclusion. The extensive mammary development found in male (Deis, '67) and female rats (Chowers and McCann, '67) bearing intrahypophyseal estrogen implants is additional evidence that secretion of prolactin is stimulated. Also, like systemically administered estrogen, these implants increase pituitary weight (Chowers and McCann, '67; Lisk, '69). The high binding affinity of the pars 
distalis for circulating ${ }^{3}$ Hestradiol as shown by counting radioactivity in pituitary extracts (Eisenfeld and Axelrod, '66) and by autoradiography (Stumpf, '68) may be one factor that facilitates direct action by estrogen.

The one consideration that could militate against the conclusion that estrogen acts directly on the hypophysis is the possible existence of vessels that carry blood from the hypophysis to the hypothalamus. Vessels with this orientation have been described in dogs and cats by Török ('64). If' estrogen implanted in the hypophysis is carried to the hypothalamus it might induce the liberation of releasing and/or inhibiting factors that could mediate the effects observed, providing there is no crossing over of estrogen or hypothalamic factors to the other side. This possibility seems unlikely and the presence of blood flow from hypophysis to hypothalamus should be confirmed.

Direct inhibition of the hypophysis. There is less agreement with respect to the capacity of estrogens to inhibit directly the secretion of gondadotropins and cause involution of their cells of origin. The regression of gonadotropes observed in this study in the pituitary lobe containing estrogen confirms a prior report by Bogdanove ('63). $\mathrm{He}$ observed a still greater involution of gonadotropic basophils, probably because his implants were left in situ for a longer period of time. However, Kanematsu and Sawyer ('63b) were unable to induce regression of postovariectomy basophils by implantation of estrogen into hypophyses of rabbits. The possible influence of diversity in species responsivity may account for these contradictory results. More likely the implantation technique employed by Kanematsu and Sawyer did not allow the development of a sufficiently high local concentration of hormone to alter the structure of basophils. The failure of Ramirez et al. ('64) to find a fall in pituitary luteinizing hormone content following intraglandular implantation of estrogen may be explained by the short duration of the experiment (12-14 days). It seems probable that a longer exposure to estrogen is required to cause involution of gonadotropes than to suppress the secretion of hormone.

Distribution of the estrogen effect. A third pertinent conclusion from this study is that spread of the estrogen effect is limited, never affecting all of one lobe of the pars distalis if the pellet is placed lateral to the midline. Further, the extent to which that lobe is affected depends on the position of the pellet. Thus, if the pellet is located at the caudal margin of the gland, little tissue will be affected.

As has been pointed out previously (Bogdanove, '63), distribution of the estrogenic effect within the pars distalis is largely determined by the pattern of blood flow in the hypothalamo-hypophyseal vascular system. The rat pars distalis is supplied by two sets of portal vessels (Landsmeer, '51; Daniel and Prichard, '56; Adams et al., '63, '65). From tortuous capillaries in the median eminence, long portal vessels run down the stalk carrying blood to the central region of the pars distalis (about $78 \%$ of the gland), the median group of vessels going to the ventral and median portions of the pars distalis and the lateral group to the rostral portion of the lateral wing. The second set of portal vessels are short; they arise from capillaries in the lower infundibular stem and supply the rostral pole of each lateral wing as well as the dorsolateral and caudal areas of the pars distalis. The area supplied by each long portal vessel appears to be sharply circumscribed. The main route of drainage within the gland is caudalward and ventrally toward veins arising along the ventrocaudal border of the gland.

In the present experiments limitation of the estrogenic effect to the side with the implanted pellet is a reflection of the bilateral nature of the hypopophyseal portal system (Adams et al., '64). Blood does not pass laterally between capillary tufts in the median eminence, between long portal vessels or between sinusoids in the gland. Therefore, when the pellet is located above one lobe of the pars distalis, distribution of estrogen is to that lobe only. Likewise from pellets within the gland, the spread was ventral lateral and caudal. It is unlikely that significant spread of estrogen 
occurred except insofar as it was carried in sinusoidal blood.

Under these circumstances one cannot expect a single estrogen implant to elicit a significant effect on the overall function of the gland since only $10 \%$ of the pars distalis is sufficient to effect some stimulation of pituitary-dependent endocrine glands in the partially hypophysectomized rat (Smith, '32). In view of this consideration, the failure of intrapituitary implants to alter size of the gland, its total hormone content, or secretion as revealed by peripheral indicators, cannot be accepted as evidence that estrogens do not act directly on the gland.

The restricted spread of estrogenic action when the hormone is implanted in the hypophysis may explain many puzzling prior observations. Thus, Kanematsu and Sawyer ('64) found that such treatment did not increase the weight of the rabbit hypophysis, although Ramirez and McCann ('64) demonstrated that it does so in the rat. The negative observation of Kanematsu and Sawyer probably resulted from the small amount of estrogen implanted and its failure to reach a significant portion of the relatively greater mass of pituitary tissue. Similar reasoning may explain also why intrapituitary implantation of estrogen in a single site failed to cause gonadal atrophy in the rabbit (Kanematsu and Sawyer, '63a) but had this effect in the rat (Chowers and McCann, '67). It may be inferred that one major factor which determines whether one can induce a generalized effect on the hypophysis by such an experimental procedure is size of the gland.

Finally, there is now general agreement that estrogen implanted in the median eminence may be carried to the pars distalis by way of the hypothalamo-hypophyseal blood vessels and exert a direct effect there (Bogdanove, '63; Palka et al., '66). In order to demonstrate the influence of hormones on secretion of hypothalamic releasing and inhibiting factors, hormones have been implanted in the region of the median eminence (Corbin and Story, '67; Motta et al., '68; Chen et al., '68, and many others). Sometimes such experiments have been controlled by concurrent implants in the hypophysis. It is clear that for such experi- mental designs to provide valid information concerning the site of hormone feedback action, the investigator must prove (a) that the hormone implanted in the median eminence fails to reach the hypophysis, (b) that a sufficiently large proportion of the hypophysis is affected by the intrapituitary implants to make possible the expected pituitary or systemic effects, or (c) that differential effects elicited by estrogen in the two sites are due to factors other than differences in hormone concentration.

It is concluded that the observations presented add additional support to the concept that the hypophysis serves as an important site for feedback action by estrogen.

\section{ACKNOWLEDGMENTS}

We express our appreciation to the following individuals for providing antisera to the hormones indicated: Dr. A. R. Midgley, Jr., prolactin and chorionic gonadotropin; Dr. S. Pek, corticotropin; and Dr. R. F. Knopf, growth hormone. Gratefully acknowledged also is the assistance of Mrs. Ya-Yen Yu in technical procedures and $\mathrm{Mr}$. W. L. Brudon in preparation of illustrations.

\section{LITERATURE CITED}

Adams, J. H., P. M. Daniel and M. M. L. Prichard 1963 The volumes of pars distalis, pars intermedia and infundibular process of the pituitary gland of the rat, with special reference to the effect of stalk section. Quart. J. Exp. Physiol, 48: 217-234.

1964 Distribution of hypophysial portal blood in the anterior lobe of the pituitary gland. Endocrinology, 75: 120-126.

- 1965 Observations on the portal circulation of the pituitary gland. Neuroendocrinology, $1: 193-213$.

Baker, B. L. 1970 Studies on hormone localization with emphasis on the hypophysis. J. Histochem. Cytochem., in press.

Baker, B. L., A. R. Midgley, Jr., B. E. Gersten and Y. Y. Yu 1969 Differentiation of growth hormone- and prolactin-containing acidophils with peroxidase-labeled antibody. Anat. Rec., 164: 163-172.

Baker, B. L., S. Pek, A. R. Midgley, Jr. and B. E. Gersten 1970 Identification of the corticotropin cell in rat hypophyses with peroxidaselabeled antibody. Anat. Rec., in press.

Birge, C. A., G. T. Peake, I. K. Mariz and W. H. Daughaday 1967 Radioimmunoassayable growth hormone in the rat pituitary gland: effects of age, sex and hormonal state. Endocrinology, 81: 195-204.

Bogdanove, E. M. 1963 Direct gonad-pituitary feedback: an analysis of effects of intracranial 
estrogenic depots on gonadotrophin secretion. Endocrinology, 73: 696-712.

Chen, C. L., J. L. Voogt and J. Meites 1968 Ef fect of median eminence implants of FSH, $\mathrm{LH}$ or prolactin on luteal function in the rat. Endocrinology, 83; 1273-1277.

Chowers, I., and S. M. McCann 1963 The effects on ACTH and gonadotrophin secretion of implants of gonadal steroids in the hypothalamo-hypophysial region. Israel Med. J., 22: 420432.

1967 Comparison of the effect of hypothalamic and pituitary implants of estrogen and testosterone on reproductive system and adrenal of female rats. Proc. Soc. Exp. Biol. Med., 124: 260-266.

Corbin, A., and J. C. Story 1967 "Internal" feedback mechanism: response of pituitary FSH and of stalk-median eminence follicle stimulating hormone-releasing factor to median eminence implants of FSH. Endocrinology, 80: 1006-1012.

Daniel, P. M., and M. M. L. Prichard 1956 Anterior pituitary necrosis. Infarction of the pars distalis produced experimentally in the rat. Quart. J. Exp. Physiol., 41: 215-229.

Davidson, J. M. and C. H. Sawyer 1961 Effects of localized intracerebral implantation of oestrogen on reproductive function in the female rabbit. Acta Endocr., 37: 385-393.

DeGroot, J, 1959 The rat forebrain in stereotaxic coordinates. N. V. Noord-Hollandsche Uitgevers Maatschappij, Amsterdam.

Deis, R. P. 1967 Mammary gland development by hypothalamic and hypophyseal estrogen implants in male rats. Acta Physiol. Lat. Amer, 17: 115-117.

Döcke, F., and G. Dörner 1965 The mechanism of the induction of ovulation by oestrogens. J. Endocr., 33: 491-499.

Eisenfeld, A. J., and J. Axelrod 1966 Effect of steroid hormones, ovariectomy, estrogen pretreatment, sex and immaturity on the distribution of ${ }^{3} \mathrm{H}$-estradiol. Endocrinology, 79: 38-42.

Flerkó, B., and J. Szentágothai 1957 Oestrogen sensitive nervous structures in the hypothalamus. Acta Endocr., 26: 121-127.

Fonzo, D., R. B. Mims and D. H. Nelson 1967 Estrogen influence on pituitary and adrenal function in the rat. Endocrinology, 81:29-33.

Kanematsu, S., and C. H. Sawyer 1963a Ef fects of intrahypothalamic and intrahypophysial estrogen implants on pituitary prolactin and lactation in the rabbit. Endocrinology, 72: 243252.

1963b Effects of hypothalamic and hy pophysical estrogen implants on pituitary gonadotrophic cells in ovariectomized rabbits. Endo. crinology, 73: 687-695.

1963c Effects of hypothalamic estrogen implants on pituitary $\mathbf{L H}$ and prolactin in rabbits. Am. J. Physiol, 205: 1073-1076.

1964 Effects of hypothalamic and hypophysial estrogen implants on pituitary and plasma LH in ovariectomized rabbits. Endocrinology, 75: 579-585.

Kitay, J. I. 1963 Pituitary-adrenal function in the rat after gonadectomy and gonadal hormone replacement. Endocrinology, 75: 253-260.

Landsmeer, J. M. F. 1951 Vessels of the rat's hypophysis. Acta Anat., 12; 82-109.

Lisk, R. D. 1960 Estrogen-sensitive centers in the hypothalamus of the rat. J. Exp. Zool., 145: 197-205.

1969 Estrogen: direct effects of hypothalamus or pituitary in relation to pituitary weight changes. Neuroendocrinology, 4: 368373.

Meites, J., and C. S. Nicoll 1966 Adenohypophysis: prolactin. Ann. Rev. Physiol., 28: 57-88.

Monroe, S. E., and A. R. Midgley, Jr. 1969 Immunofuorescent localization of rat luteinizing hormone. Proc. Soc. Exp. Biol. Med., 130: 151156.

Motta, M., F. Fraschini, G. Giuliani and L. Martini 1968 The central nervous system, estrogen and puberty. Endocrinology, 83: 1101-1107.

Nakane, P. 1970 Localization of pituitary hormones with immumo-enzyme histochemistry. J. Histochem. Cytochem., in press.

Nakane, P. K., and G. B. Pierce, Jr, 1967 Enzyme-labeled antibodies for the light and electron microscopic localization of tissue antigens. J. Cell Biol., 33: 307-318.

Nicoll, C. S., and J. Meites 1962 Estrogen stimulation of prolactin production by rat adenohypophysis in vitro. Endocrinology, 70: 272-277.

Palka, Y. S., V. D. Ramirez and C. H. Sawyer 1966 Distribution and biological effects of tritiated estradiol implanted in the hypothalamohypophysial region of female rats, Endocrinology, $78: 487-499$.

Purves, H. D. 1961 Morphoiogy of the hypophysis related to its function. In: Sex and Internal Secretions, third edition. W. C. Young, ed. Williams and Wilkins Co., vol. 1, pp. 161-239.

Ramirez, V. D., R. M. Abrams and S. M. McCann 1964 Effect of estradiol implants in the hypothalamo-hypophysial region of the rat on the secretion of luteinizing hormone. Endocrinology, 75: 243-248.

Ramirez, V. D., and S. M. McCann 1964 Induction of prolactin secretion by implants of estrogen into the hypothalamo-hypophysial region of female rats. Endocrinology, 75: 206-214.

Richard, R. 1965 Estrogen effects of pituitaryadrenal function via the hypothalamus and hypophysis. Neuroendocrinology, 1: 322-332.

Smith, P. E. 1932 The secretory capacity of the anterior hypophysis as evidenced by the effect of partial hypophysectomies in rats. Anat. Rec., 52: 191-208.

Stumpf, W. E. 1968 Cellular and subcellular ${ }^{3}$ H-estradiol Iocalization in the pituitary by autoradiography. Z. Zellforsch., 92: 23-33.

Török, B. 1964 Structure of the vascular connections of the hypothalamo-hypophysial region. Acta Anat., 59: 84-99. 
PLATES 
All photographs are of immunochemically stained preparations which were cut in a dorsoventral plane.

\section{PLATE 1}

\section{EXPLANATION OF FIGURES}

1 Hypophysis stained for prolactin cells. The left lobe of the pars distalis contained a cholesterol pellet ( $P$ ) which was implanted stercotaxically 14 days after ovariectomy and left in situ for 14 more days. The dark spots in the pars distalis are prolactin cells. $\times 16$.

2 Hypophysis stained for prolactin cells, showing the area lateral to the pellet indicated in figure 1 . The dark prolactin cells are exceedingly small as a result of ovariectomy. $\times 100$. Inset; an enlarged view of a typical prolactin cell in this section. $\times 1000$.

3 Hypophysis stained for prolactin cells. The left lobe of the pars distalis contained an estradiol pellet (P) which was jmplanted stereotaxically 14 days after ovariectomy and left in situ for 14 additional days. The ipsilateral lobe is enlarged. The dark spots are enlarged prolactin cells. The zone of estrogen stimulation stops at the broken line and does not reach the midline. $\times 16$.

4 Hypophysis stained for prolactin cells, showing the contralateral area indicated on figure 3. Prolactin cells (arrows) are exceedingly small. $\times 100$. Inset: a typical small prolactin cell from this section. $\times 1000$.

5 Hypophysis stained for prolactin cells, showing area lateral to the estrogen pellet, as indicated in figure 3. Prolactin cells are generally enlarged. $\times 100$. Inset: a typical prolactin cell from this area showing large cytoplasmic granules and hypertrophied Golgi region. $\times 1000$.

6 Hypophysis stained for prolactin cells, illustrating area medial to the estrogen pellet as indicated in figure 3 . Prolactin cells in the lower portion of the field have not been stimulated by estrogen. $\times 100$. 


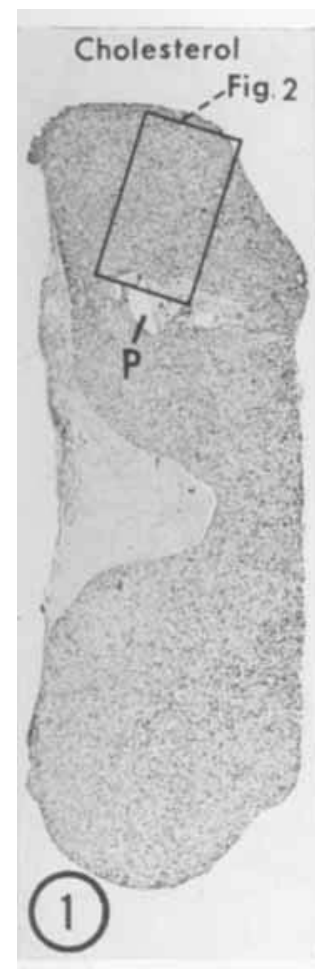

\section{Estradiol}

Fig.5、
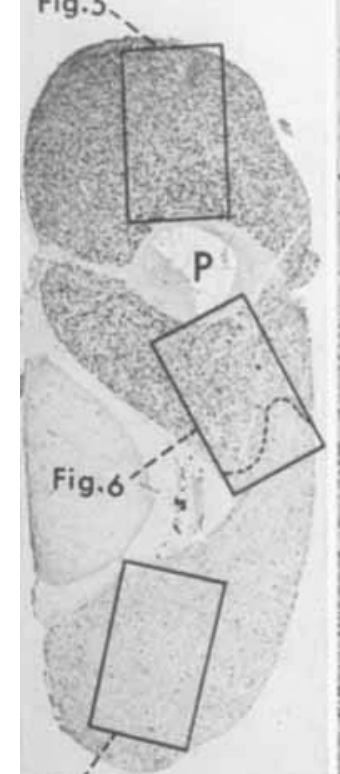

Fig. 4

(3)

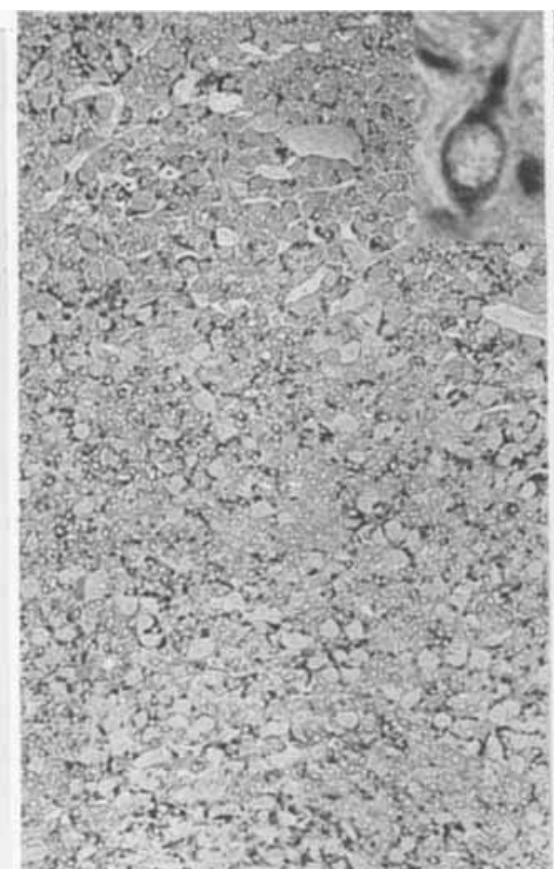

(2) $p$ pere

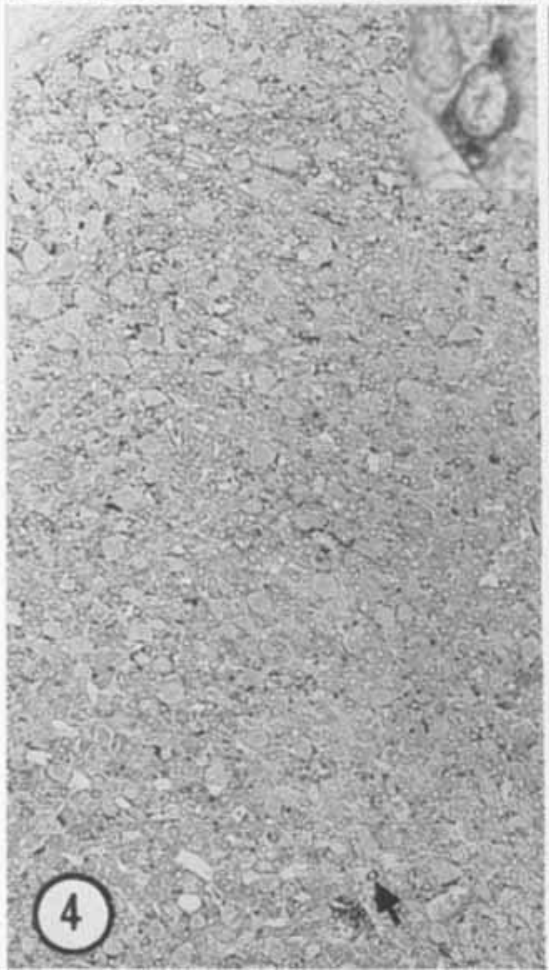

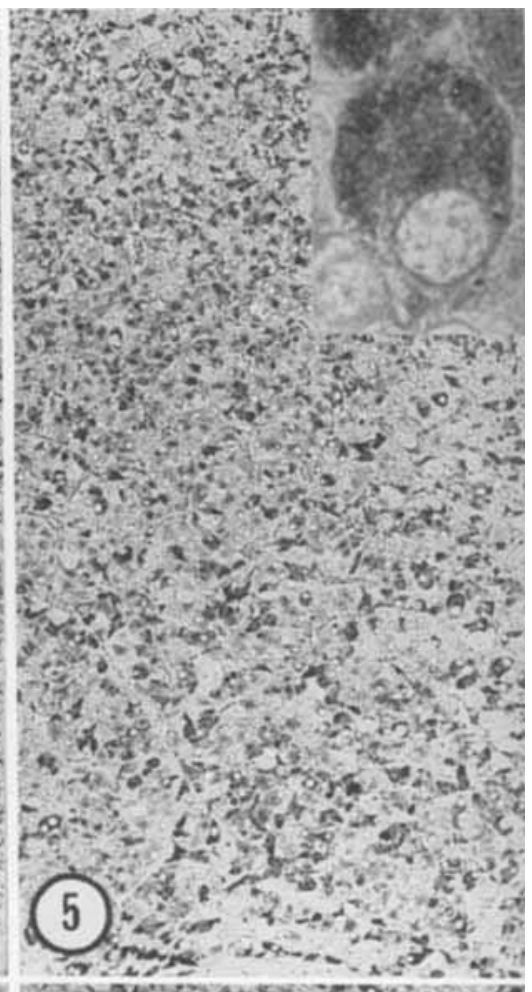

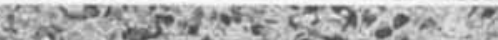
201.35

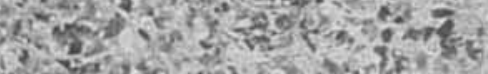

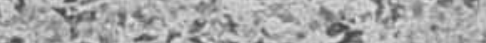

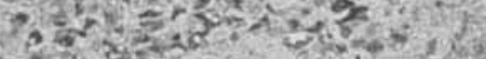

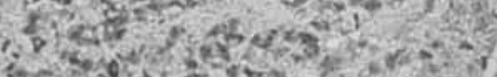

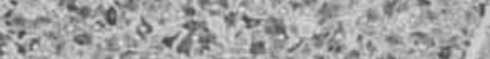

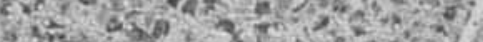

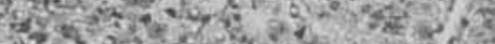

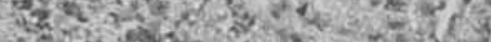

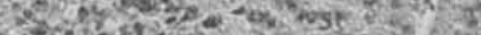

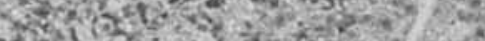

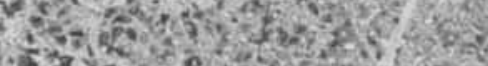

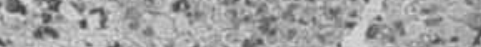

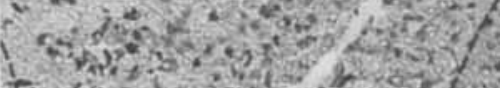

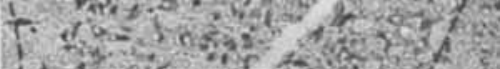

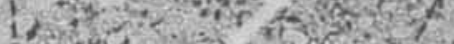

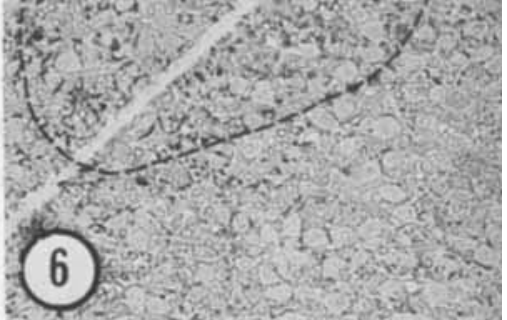


PLATE 2

\section{EXPLANATION OF FIGURES}

7 Hypophysis stained for prolactin cells. The left lobe contained an estrogen pellet (P) placed by the retropharyngeal approach 29 days after ovariectomy and left in place an additional 11 days. The region of prolactin cell enlargement (dark spots) is restricted to the region of the pellet. $\times 16$.

8 Hypophysis stained for prolactin cells illustrating area indicated in figure 7. Prolactin cells are stimulated lateral to the pellet (P) as far as the broken line. $\times 100$

9 Hypophysis stained for prolactin cells illustrating area indicated in figure 7. Prolactin cells are stimulated medial to the pellet as far as the broken line. $\times 100$.

10. Hypophysis stained for prolactin cells, In this case an estrogen pellet was inadvertently implanted stereotaxically into the hypothalamus on the left side. The implantation occurred 14 days after ovariectomy and the pellet remained in place an additional 14 days. Prolactin cells (dark spots) are enlarged in the ipsilateral lobe of the pars distalis (below), the effect extending across the midline to the broken line. $\times 16$.

11 Hypophysis stained for prolactin cells, illustrating the area of the contralateral lobe indicated in figure 10. The prolactin cells (dark spot, arrow) are small as a result of ovariectomy. $\times 100$.

12 Hypophysis stained for prolactin cells, illustrating the ipsilateral side in the area indicated in figure 10. Prolactin cells are generally enlarged. $\times 100$. 


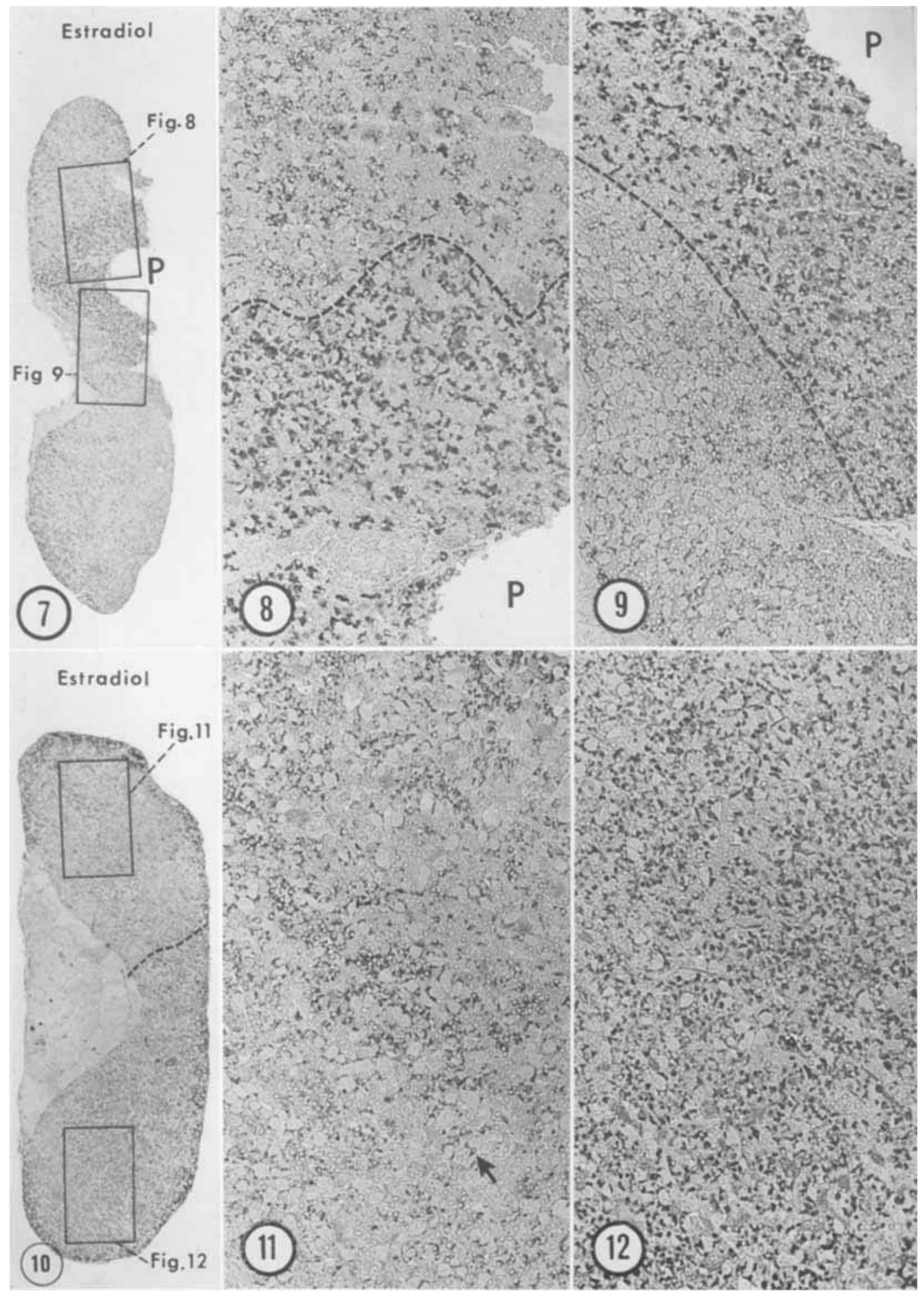




\section{PLATE 3}

EXPLANATION OF FIGURES

13 Hypophysis stained for growth hormone cells. The left lobe contained a cholesterol pellet ( $P$ ) which was implanted stereotaxically 14 days after ovariectomy and left in place for an additional 14 days. Growth hormone cells (dark spots) are uniformly distributed in both lobes of the pars distalis. $\times 16$.

14 Hypophysis stained for growth hormane cells and illustrating the area indicated on figure 13. Cholesterol did not cause any change in these cells. $\times 100$. Jnset; an enlarged view of typical growth hormone cells. $\times 1000$.

15 Hypophysis stained for growth hormone cells (same gland as illustrated in fig. 3 ). The left lobe of the pars distalis contained an estrogen pellet (P) which was implanted stereotaxically 14 days after ovariectomy and left in place for an additional 14 days. $\times 16$.

16 Hypophysis stained for growth hormone cells showing the area indicated in figure 15 in the contralateral lobe of the pars distalis. $\times 100$ Inset: an enlargement of some typical growth hormone cells from this area. They are not significantly different from those in the cholesterol-implanted gland (fig. 14), $\times 1000$.

17 Hypophysis stained for growth hormone cells, and illustrating an area lateral to the estrogen pellet as indicated on figure 15. Growth hormone cells are somewhat smaller and have denser cytoplasm than those in the contralateral lobe (fig. 16). The growth hormone cells are more dispersed because of the enlarged intervening prolactin cells. $\times 100$. Inset: individual grawth hormone cells showing reduction in size due to estrogen action. $\times 1000$.

18 Hypophysis stained for growth hormone cells, showing the area medial to the estrogen pellet as indicated on figure 15 . The response is similar to that lateral to the pellet. $\times 1000$. 


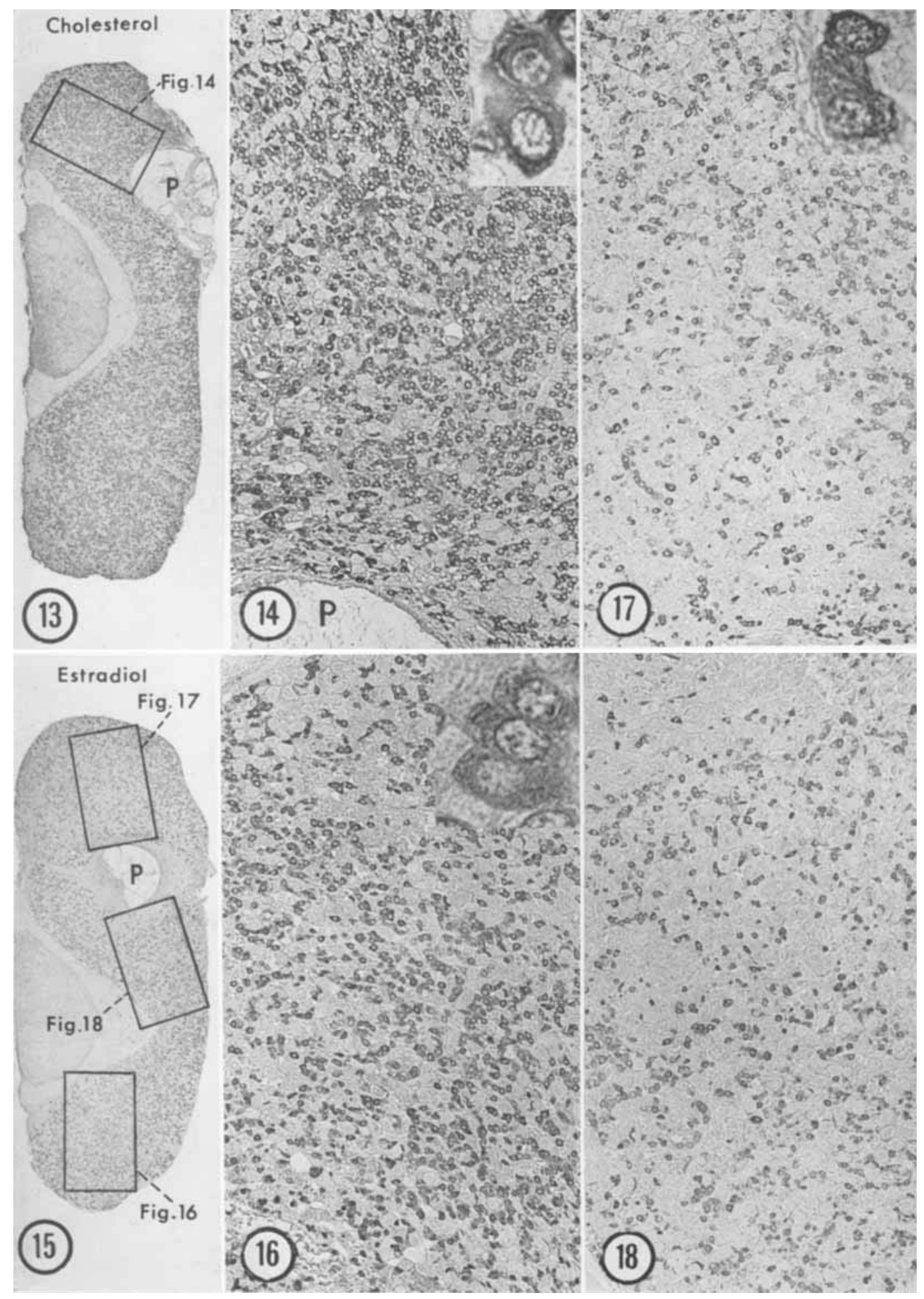


19 Hypophysis stained for corticotropin cells. The left lobe contained a cholesterol pellet which was implanted 14 days after ovariectomy and left in place 14 additional days. The corticotropin cells (dark spots) have a similar distribution in both lobes of the pars distalis. $\times 16$.

20 Hypophysis stained for corticotropin cells, illustrating the area indicated in figure 19. The corticotropin cclls are black. $\times 100$. Inset: an enlarged corticotropin cell. $\times 1000$.

21 Hypophysis stained for corticotropin cells. The left lobe of the pars distalis contained an estrogen pellet (P) which was implanted 14 davs after ovaricctomy and left in place for another 14 days. $\times 100$.

22 Hypophysis stained for corticotropin cells, showing the area in the contralateral lobe of the pars distalis as indicated on figure 21. The size and distribution of corticotropin cells are similar here and in the cholesterol-implanted gland (fig. 20), $\times 100$. Inset: a typical corticotropin cell from this area, $\times 1000$.

23 Hypophysis stained for corticotropin cells, showing the area lateral to the estrogen pellet as indicated in figure 21. As compared with the contralateral side, corticotropin cells arc unchanged in size but more widely dispersed owing to enlargement of prolactin cells. $\times 100$. Inset: a typical corticotropin cell from this area. $\times 1000$.

24 Hypophysis stained for corticotropin cells, illustrating an area medial to the estrogen pellet as indicated in figure 21. Close to the pellet (upper section) the picture is somewhat similar to that lateral to the pellet (fig. 23). $\times 100$. 


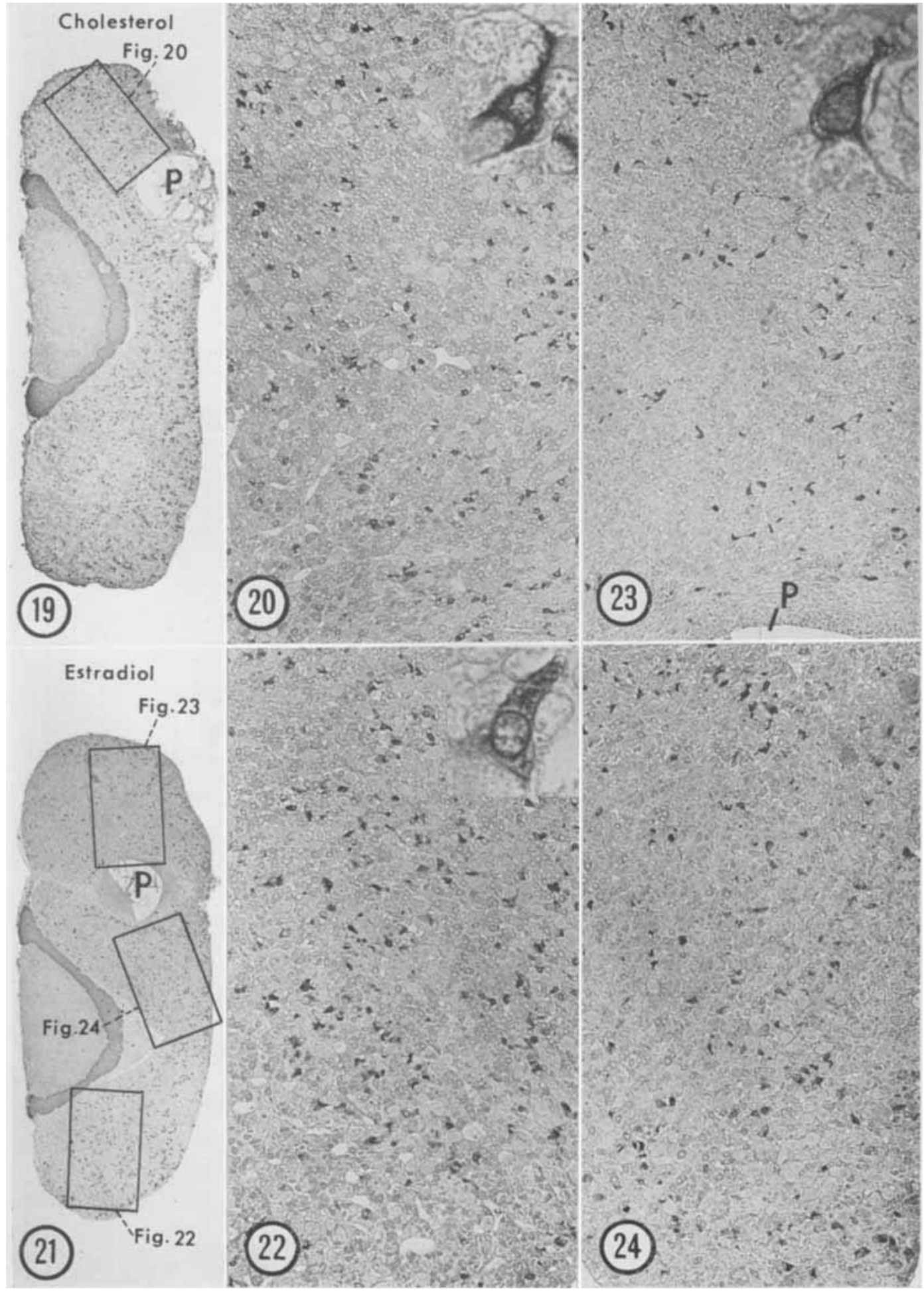




\section{PLATE 5}

EXPLANATION OF FIGURES

25 Hypophysis stained for gonadotropes. The left lobe of the pars distalis contained a cholesterol pellet ( $P$ ) which was implanted stereotaxically 14 days after ovariectomy and left in place for an additional 14 days (same gland as illustrated in figs. 13 and 19). In this specimen gonadotropes (dark spots) seem somewhat fewer dorsolateral to the pellet. This was not the case in other cholesterol-implanted glands. $\times 16$.

26 Hypophysis stained for gonadotropes and jllustrating the area indicated in figure 25. $\times 100$. Inset: a typical gonadotrope. $\times 1000$.

27 Hypophysis stained for gonadotropes (same gland as illustrated in figs. 3,15 and 21 ). The left lobe of the pars distalis contained an estrogen pellet (P) which was implanted stereotaxically 14 days after ovariectomy and left in place for an additional 14 days. $\times 16$.

28 Hypophysis stained for gonadotropes, showing the area of the contra lateral lobe as indicated in figure 27. Gonadotropes are somewhat larger then in the cholesterol-implanted gland (fig. 26). $\times 100$. Inset: a large gonadotrope from this field. $\times 1000$.

29 Hypophysis stained for gonadotropes showing the area lateral to the estrogen pellet indicated in figure 27. Gonadotropes are fewer, smaller, and have denser cytoplasm. $\times 100$. Inset: a typical gonadotrope from this field, $\times 1000$.

30 Hypophysis stained for gonadotropes showing the area medial to the estrogen pellet as indicated in figure 27 . The changes described for figure 29 occur only near the pellet. $\times 100$. 


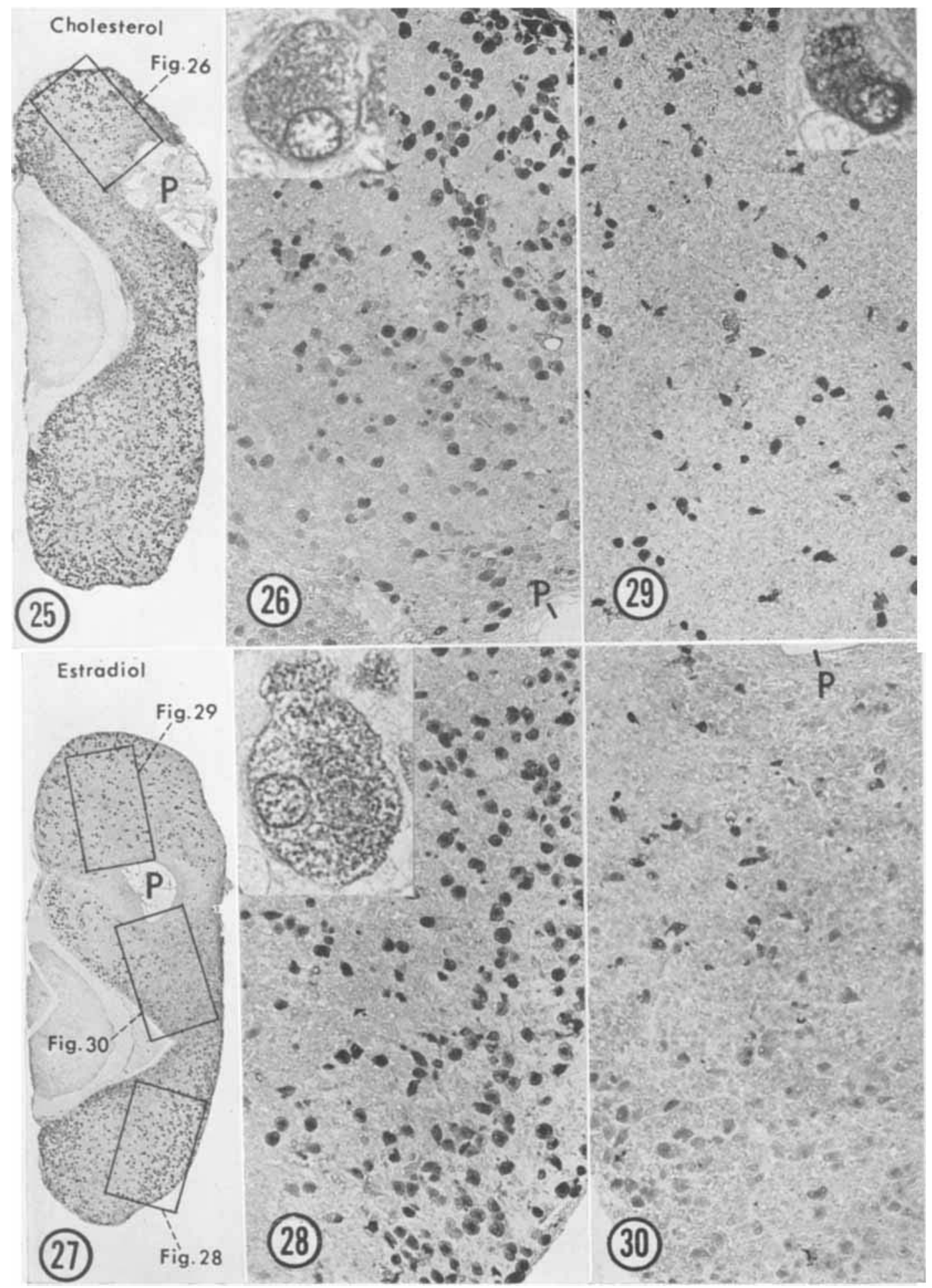

